\title{
Conhecimento sobre plantas medicinais por mulheres em processo de envelhecimento
}

\section{Knowledge about medicinal plants by women in aging process}

\author{
Morgana Schiavo $^{1}$, Gabriela Tassotti Gelatti ${ }^{2}$, Karla Renata de Oliveira ${ }^{3}$, Vanessa Adelina Casali \\ Bandeira $^{4}$, Christiane de Fátima Colet $^{5}$
}

\begin{abstract}
Resumo
Este estudo objetiva avaliar o conhecimento quanto à espécie e indicação de plantas medicinais utilizadas por mulheres em processo de envelhecimento. Trata-se de estudo transversal, quantitativo, vinculado à pesquisa "Estudo multidimensional de mulheres no processo de envelhecimento". A coleta de dados foi realizada nas residências das participantes, através da aplicação de um questionário e coleta do material vegetal para a identificação botânica. Entre as 84 participantes, $94 \%$ faziam uso de planta medicinal para tratar doenças e $56 \%$ como aromatizante no chimarrão, bebida característica da região Sul, preparada com erva mate moída e água quente. Foram identificadas 148 plantas medicinais de 23 famílias e 47 espécies diferentes. Destas, 20 espécies foram classificadas como adequadas quanto à indicação, conforme a literatura pesquisada, 12 espécies estavam parcialmente adequadas, 10 espécies estavam inadequadas e 5 espécies não foram encontradas nas bibliografias. Constatou-se que muitas espécies estão em desacordo com a literatura quanto ao uso relatado pelas participantes, o que reforça a necessidade de orientação para essas usuárias do serviço público de saúde, relativas à indicação e a toxicidade das plantas medicinais.
\end{abstract}

Palavras chave: Climatério. Estratégia saúde da família. Etnobotânica. Plantas medicinais. Saúde da mulher.

\begin{abstract}
This study objective was to evaluate the knowledge on the species and indication of medicinal plants used by women in the aging process. It is a cross-sectional, quantitative study, linked to research "multidimensional study of women in the aging process." Data collection was carried out in the homes of the participants, by applying a questionnaire and the collection of plant material for botanical identification. The 84 participants, $94 \%$ were using of medicinal plant to treat diseases and $56 \%$ in mate as flavoring that is a characteristic drink of the southern region, prepared with ground grass mate and hot water. It was realized the identification of 148 medicinal plants of 23 families and 47 different species have been identified. Of these, 20 species were appropriate according to the literature, 12 species were partially adequate, 10 species were inadequate and 5 species were found in the surveyed bibliographies. It was found that many species are at odds with the literature regarding the use indicated by participants, which indicates the need for guidance to those users of the public health service regarding the use and toxicity of medicinal plants.
\end{abstract}

Keyword: Climacteric. Ethnobotany. Family health strategy. Plants medicinal. Women's health.

\footnotetext{
${ }^{1}$ Farmacêutica pela Universidade Regional do Noroeste do Estado do Rio Grande do Sul. Ijuí, Rio Grande do Sul, Brasil.

${ }^{2}$ Mestranda em Atenção Integral à Saúde pela Universidade de Cruz Alta em associação com a Universidade Regional do Noroeste do Estado do Rio Grande do Sul; Cruz Alta, Rio Grande do Sul, Brasil.

${ }^{3}$ Mestrado em Ciências Biológicas e Bioquí mica pela Universidade Federal do Rio Grande do Sul. Departamento de Ciências da Vida, Universidade Regional do Noroeste do Estado do Rio Grande do Sul; Ijuí, Rio Grande do Sul, Brasil.

${ }^{4}$ Mestranda em Atenção Integral à Saúde pela Universidade de Cruz Alta em associação com a Universidade Regional do Noroeste do Estado do Rio Grande do Sul; Cruz Alta, Rio Grande do Sul, Brasil. E-mail: vanessa.acbandeira@yahoo.com.br

${ }^{5}$ Doutorado em Ciências Farmacêuticas pela Universidade Federal do Rio Grande do Sul. Universidade Regional do Noroeste do Estado do Rio Grande do Sul; Ijuí, Rio Grande do Sul, Brasil.
} 


\section{Introdução}

O uso de plantas com fins medicinais está relacionado aos primórdios da medicina, resultado do acúmulo secular de conhecimentos empíricos sobre a ação dos vegetais por diversos grupos étnicos (BRASIL, 2006b; SIMÕES et al., 2007). Nos últimos anos há interesse crescente e renovado no uso de terapias complementares, especialmente vegetais. Esse crescimento está relacionado a fatores entre os quais se destacam a decepção com os resultados obtidos em tratamentos com a medicina convencional, os efeitos indesejáveis e prejuízos causados pelo uso abusivo ou incorreto de medicamentos, as dificuldades de acesso por parte da população aos medicamentos e a crença popular de que o natural é inofensivo (RATES, 2001). Além disso, este aumento está relacionado com legislações publicadas com incentivo de acesso, promoção do uso e de pesquisas sobre plantas medicinais; como a Política Nacional de Plantas Medicinais e Fitoterápicos (PNPMF) e Política Nacional de Práticas Integrativas e Complementares (PNPIC) (BRASIL, 2006a, 2006b).

Essas políticas incentivam a inserção de plantas medicinais e fitoterápicos no Sistema Único de Saúde (SUS) e fornecem subsídios para o uso adequado. A divulgação de informações científicas relacionadas ao uso de plantas medicinais é fundamental para a sociedade, uma vez que o uso dessas é frequente na população, com percentual de uso na população adulta entre $57 \%$ a $85 \%$ conforme estudos realizados em diferentes regiões do Brasil (LIMA; PIRES; VIEIRA, 2014; MATOS; MATOS; BRITO, 2008; OLIVEIRA; LUCENA, 2015). Além disso, as informações relacionadas ao uso de plantas medicinais são provenientes do conhecimento popular, fornecidas principalmente por familiares, ou seja, transmitidas de geração para geração (DELL'ANTONIO et al., 2015; PEREIRA et al., 2015; ROSA et al., 2014).

Evidencia-se nos estudos nacionais, maior frequência de uso de plantas medicinais entre as mulheres, por assumirem geralmente as responsabilidades relacionadas às práticas de cuidado no contexto familiar e por serem receptoras dos conhecimentos tradicionais repassados entre as gerações (OLIVEIRA; LUCENA, 2015; PEREIRA et al., 2015; SOUZA et al., 2011). Nesse contexto, cultivam, manipulam e utilizam plantas medicinais para os mais distintos sintomas e tornam-se, assim, referência no cuidado da saúde familiar e comunitária (HOGA, 2008; KARAM, 2004; LIMA et al., 2014).
Além disso, estudos anteriores apontam maior uso e conhecimento de plantas medicinais nas faixas etárias mais avançadas, principalmente entre as mulheres com idade superior a 30 anos (LIMA et al., 2014; OLIVEIRA; LUCENA, 2015; PEREIRA et al., 2015). Ressalta-se que a presença de doenças se acentua com o envelhecimento e o uso de plantas medicinais representa um tratamento complementar ou alternativo (BRASIL, 2008). No processo de envelhecimento feminino, destaca-se o período do climatério, no qual as mulheres ampliam seus cuidados com a saúde e buscam alternativas para o tratamento dos sintomas relacionados como os "fogachos", entre os quais incluise o uso de plantas e fitoestrógenos por apresentarem menos efeitos adversos que os hormônios sintéticos (GONÇALVES et al., 2011; ROCHA; ROCHA, 2010).

Com base nessas considerações e diante da necessidade de conhecer as características de cuidado durante o processo de envelhecimento, entre esses o uso de plantas medicinais, o presente estudo tem como objetivo avaliar o conhecimento quanto à espécie e indicação de plantas medicinais utilizadas por mulheres em processo de envelhecimento.

\section{Material e Método}

Trata-se de um estudo transversal e quantitativo, realizado com mulheres adstritas a duas unidades de Estratégias Saúde da Família (ESF) do município de Ijuí/RS, participantes da pesquisa institucional "Estudo multidimensional de mulheres no processo de envelhecimento" da Universidade Regional do Noroeste do Estado do Rio Grande do Sul (UNIJUÍ), aprovada pelo Comitê de Ética em Pesquisa da UNIJUÍ sob o Parecer Consubstanciado $n^{\circ} 864.988 / 2014$. A seleção das mulheres neste projeto deu-se de forma intencional selecionando todas as mulheres que contemplam os critérios de inclusão do estudo.

$\mathrm{Na}$ população do estudo, foram incluídas mulheres com idade entre 35 e 65 anos, adstritas as ESFs do município de Ijuí/RS. Os dados foram coletados através da aplicação de um questionário na residência das participantes, no período de dezembro de 2013 a fevereiro de 2014. O questionário era subdividido em três partes, a primeira com questões socioeconômicas como idade, escolaridade, estado civil, renda e profissão; a segunda referia-se à utilização das plantas medicinais com abordagem sobre o consumo e aceitação das mesmas; a terceira parte era relacionada às plantas que eram utilizadas pelas entrevistadas; e incluía questões como nome popular, modo de preparo 
do chá (infusão, decocção e maceração), parte da planta utilizada e indicação da mesma.

Além do questionário supracitado, foi realizada coleta do material vegetal nas residências para a identificação botânica das espécies vegetais. As amostras coletadas continham partes como folhas, flores e frutos, dando prioridade a ramos adultos. Depois de coletadas realizou-se a exsicata das plantas, conforme citado na metodologia de Mentz e Bordignon (2004). Para a identificação das espécies e/ou famílias botânicas, as amostras foram analisadas por uma engenheira agrônoma.

As plantas medicinais identificadas foram avaliadas quanto ao uso farmacológico e indicação de uso conforme: "Formulário de Fitoterápicos da Farmacopeia Brasileira" (ANVISA, 2011); "Farmacognosia: da planta ao medicamento"(SIMÕES et al., 2007); "Plantas Medicinais no Brasil: nativas e exóticas" (LORENZI; MATOS, 2008); "Tratado das Plantas Medicinais: mineiras, nativas e cultivadas" (GRANDI, 2014).

As entrevistadas relataram a finalidade do uso das plantas citadas, e através desta informação foram classificadas as indicações autorreferidas em: de acordo com a literatura (AC), desacordo com a literatura (D), parcialmente adequado (PA) ou não encontrada na literatura pesquisada (NA).

Para a construção do banco de dados e análise foi utilizado o Software Statistical Package for the Social Sciences (SPSS) (versão 18.0), para a análise descritiva das variáveis qualitativas empregou-se frequência relativa e absoluta; para as variáveis quantitativas, medida de tendência central (média) e de dispersão (desvio-padrão, valor máximo e mínimo).

Esta pesquisa se trata de um subprojeto vinculado ao projeto institucional supracitado e foi aprovado pelo Comitê de Ética em Pesquisa da UNIJUÍ sob Parecer Consubstanciado $\mathrm{n}^{\circ}$ 61294/2012, CCAE 02711712.9.0000.5350.

\section{Resultados}

Foram entrevistadas 84 mulheres, com idade média de 51,32 \pm 7,9 anos. Entre as características socioeconômicas verificou-se maior frequência da faixa etária entre 56 a 65 anos (36,9\%); quanto à escolaridade, $45,2 \%$ das participantes não concluíram o ensino fundamental; $57,1 \%$ das mulheres eram casadas, $42,8 \%$ relatou receber entre um e dois salários mínimos e em relação à profissão destacou-se a "do lar" $(44,1 \%)$, conforme apresentadas na Tabela 1.
O uso de plantas medicinais foi relatado por $94 \%$ das entrevistadas e $50 \%$ acreditavam que essas não fazem mal a sua saúde. Além disso, $56 \%$ das entrevistadas referiram utilizar algum tipo de planta medicinal como aromatizante no chimarrão, bebida preparada com erva mate moída e água quente. Entre os usuários de plantas medicinais, a maioria (81\%) mencionou que o conhecimento sobre o uso dessas foi repassado por familiares, de geração em geração. Quanto a aquisição das plantas, 54,4\% informaram que cultivam em sua residência (Tabela 2).

Quando questionadas sobre o modo de preparo dos chás, a infusão ( $80 \%$ ) foi o mais citado pelas entrevistadas, seguida pela decocção $(14,86 \%)$ e maceração $(5,14 \%)$. A parte vegetal mais utilizada por esta população foram as folhas (72\%) (Tabela 3). Quanto à utilização das plantas medicinais, foram citadas 70 espécies distintas, totalizando 322 plantas. Entre as plantas citadas, foram coletadas e identificadas 148 plantas medicinais de 23 famílias e 47 espécies diferentes. As plantas mais utilizadas diariamente foram a Achyrocline satureioides (Lam) DC. (Marcela, $\mathrm{n}=17$ ); Matricaria recutita (Camomila ou maçanilha, $\mathrm{n}=14$ ), Plectranthus barbatus Andrews, (Falso boldo ou Boldo brasileiro, $\mathrm{n}=11$ ), Mentha $x$ piperita var. citrata, (Hortelã pimenta, $\mathrm{n}=9$ ). As famílias botânicas mais representativas foram Asteraceae (onze espécies), Lamiaceae (seis espécies), Myrtaceae (quatro espécies). Estas informações estão apresentadas na Tabela 3.

Dentre as plantas citadas na Tabela 3 , cinco (10,7\%) espécies não foram encontradas nas bibliografias pesquisadas, $20(42,5 \%)$ espécies estão $\mathrm{AC}$ com a literatura, $12(25,5 \%)$ espécies estão PA e $10(21,3 \%)$ espécies estão em D. Além disso, todas as plantas medicinais foram citadas pelas entrevistadas pelo nome popular correto.

\section{Discussão}

A média de idade das entrevistadas foi superior a 50 anos, dado semelhante a Battisti et al. (2013) em estudo quali-quantitativo sobre as plantas medicinais utilizadas por moradores de Palmeira das Missões/ RS, no qual $90 \%$ eram do sexo feminino, a maioria com idade superior a 55 anos. Assim como, no estudo de Pilla, Amorozo e Furlan (2006), nas residências de Mogi-Mirim/SP, que realizou um inventário das plantas medicinais conhecidas e utilizadas pelos moradores daquele local e observaram que $88 \%$ dos participantes eram mulheres, com idade entre 21 e 69 
Tabela 1 - Características socioeconômicas das mulheres climatéricas atendidas nas ESFs VII e VIII do município de Ijuí/RS, 2014. N= 84 .

\begin{tabular}{|c|c|c|}
\hline Características socioeconômicas & $\mathbf{N}$ & $\%$ \\
\hline \multicolumn{3}{|l|}{ Faixa etária } \\
\hline 35 a 45 anos & 23 & 27,4 \\
\hline 46 a 55 anos & 30 & 35,7 \\
\hline 56 a 65 anos & 31 & 36,9 \\
\hline \multicolumn{3}{|l|}{ Escolaridade } \\
\hline Ensino Fundamental Incompleto & 38 & 45,2 \\
\hline Ensino Médio Completo & 16 & 19,1 \\
\hline Ensino Fundamental Completo & 12 & 14,3 \\
\hline Ensino Médio Incompleto & 7 & 8,3 \\
\hline Ensino Superior Completo & 7 & 8,3 \\
\hline Ensino Superior Incompleto & 3 & 3,6 \\
\hline Analfabeto & 1 & 1,2 \\
\hline \multicolumn{3}{|l|}{ Estado civil } \\
\hline Casada & 48 & 57,1 \\
\hline Solteira & 12 & 14,3 \\
\hline Divorciada & 12 & 14,3 \\
\hline Viúva & 4 & 4,8 \\
\hline Outro & 8 & 9,5 \\
\hline \multicolumn{3}{|l|}{ Renda } \\
\hline De 1 a 2 Salários Mínimos & 36 & 42,8 \\
\hline 1 Salário Mínimo & 21 & 25 \\
\hline Acima de 2 Salários Mínimos & 21 & 25 \\
\hline Não respondeu & 1 & 1,2 \\
\hline Abaixo de 1 Salário Mínimo & 3 & 3,6 \\
\hline Não possui renda & 2 & 2,4 \\
\hline \multicolumn{3}{|l|}{ Profissão } \\
\hline Do lar & 37 & 44,1 \\
\hline Doméstica & 10 & 11,9 \\
\hline Agente Comunitária de Saúde & 5 & 5,9 \\
\hline Outras $^{1}$ & 32 & 38,1 \\
\hline
\end{tabular}

${ }^{1}$ Aposentada, autônoma, babá, vendedora, professora, cuidadora.

Fonte: Autores.

anos; e de Pereira et al. (2015) realizado em Picos/PI no qual $49 \%$ tinham entre 31 e 55 anos. Esses dados podem ser justificados pela conduta feminina, relativa a este grupo ter mais preocupação com a saúde e estar mais atenta à sintomatologia das doenças do que os homens (BERTOLDI et al., 2004).

Quanto à escolaridade, a maioria das mulheres apresentou ensino fundamental incompleto (Tabela 1), assim como observado no estudo de Pilla, Amorozo e Furlan (2006), no qual 66\% dos participantes não completaram o ensino fundamental. Lima, Pires e Vieira (2014) em estudo realizado com a população do distrito de União Bandeirante-RO observaram que o conhecimento sobre as plantas medicinais apresenta uma tendência a diminuir com o aumento do nível de escolaridade.

Tabela 2 - Indicação e aquisição de plantas medicinais por mulheres climatéricas atendidas nas ESFs VII e VIII do município de Ijuí/RS. 2014. N=79.

\begin{tabular}{lcc}
\hline & N & $\mathbf{\%}$ \\
\hline Quem indicou as plantas medicinais & & \\
\hline Família & 64 & 81 \\
Vizinhos & 7 & 8,9 \\
Pastoral da saúde & 6 & 7,6 \\
Outros & 2 & 2,5 \\
\hline Onde adquire as plantas medicinais & & \\
\hline Plantações caseiras & 43 & 54,4 \\
Supermercado & 24 & 30,4 \\
Farmácia & 8 & 10,1 \\
Vizinhos & 3 & 3,8 \\
Pastorais & 1 & 1,3 \\
\hline
\end{tabular}

Fonte: Autores.

Mais da metade das participantes do presente estudo eram casadas (Tabela 1), semelhante à Petry e Roman Júnior (2012) cujo objetivo foi avaliar a aceitação de um programa de fitoterápicos e plantas medicinais no SUS, em Três Passos/RS, 68\% dos participantes relataram serem casados/amasiados. Este dado também está relacionado aos critérios de inclusão deste estudo, que foram mulheres, entre $35 \mathrm{e}$ 65 anos e a maioria delas já se encontra casada nesta fase da vida.

Quanto à renda das entrevistadas, predominou as que recebem de um a dois salários mínimos (Tabela $1)$, assim como no estudo realizado por Battisti et al. (2013), no qual 88\% dos entrevistados apresentam renda inferior a três salários mínimos. As plantas medicinais apresentam baixo custo e nesse estudo observou-se alta frequência de cultivo domiciliar das plantas para consumo. Muitas pessoas optam por usar as plantas medicinais por falta de acesso, dificuldade de atendimento nos serviços de saúde ou por falta de 
recursos financeiros para adquirir os medicamentos (BADKE et al., 2011). Com relação à profissão, assim como no presente estudo, Pilla, Amorozo e Furlan (2006) verificaram que $56,8 \%$ das mulheres entrevistadas eram donas de casa e $18,2 \%$ trabalhavam no comércio local. Dado também relacionado às características econômicas das entrevistadas e os critérios de inclusão no estudo embora, tenha se buscado horários alternativos para realização das entrevistas com o intuito de incluir também as mulheres que trabalham.

Neste estudo, o uso de plantas medicinais relatado pela maioria das mulheres foi elevado, o que também foi observado em Teutônia/RS por Schwambach e Amador (2007), no qual se verificou que o uso de plantas medicinais é uma prática entre $92,9 \%$ dos entrevistados; porém, cabe ressaltar que o estudo citado não foi realizado exclusivamente com mulheres, mas com aquele responsável por cuidar de saúde da família, sendo a maioria mulheres.

Mais da metade das mulheres que usavam plantas relataram utilizar algum tipo de planta medicinal como aromatizante do chimarrão. O chimarrão é uma bebida característica do Rio Grande do Sul, preparado com erva-mate moída, na qual é acrescida água quente; as plantas adicionadas são geralmente utilizadas para aromatizar essa preparação. Foram citadas Matricaria recutita (camomila), Aloysia triphylla Royle (cidró), Foeniculum vulgare Mill (erva doce), Mentha x piperita var. citrata (Ehrh.) Briq. (hortelã pimenta) entre outras. Este resultado foi semelhante ao encontrado por Battisti et al. (2013), sendo que nesse as plantas adicionadas ao chimarrão foram: Chamomilla recutita (L.) Rauschert. (camomila); Pimpinella anisum L. (erva doce); Equisetum hyemale L. (cavalinha); Illicium verum Hook. (anis estrelado) e Aloysia citrodora Ortega ex. Pers. (cidró). Estes mesmos autores destacam que muitas vezes é adicionada espécie vegetal ao chimarrão, pois é uma bebida típica do Sul do Brasil preparada com a erva mate (Ilex paraguariensis A.St.Hil.) por infusão. Entretanto, o hábito de consumir plantas no chimarrão deve ser analisado com cautela, pois se deve considerar o modo de preparo de cada planta, que nem sempre é indicado por infusão, como no chimarrão, além do que podem ocorrer interações quando utilizadas plantas em associação, o que provavelmente não é do conhecimento das entrevistadas. No presente estudo as mulheres que referiram o uso de plantas medicinais como aromatizantes no chimarrão não foram questionadas sobre o conhecimento da indicação terapêutica dessas, tratando-se de uma limitação.

A maioria das entrevistadas informou que $o$ conhecimento sobre o uso de plantas medicinais foi repassado por familiares (Tabela 2), assim como no estudo de Alves e Povh (2013) realizado na comunidade rural de Santa Rita, no município de Ituiutaba/MG, no qual $90 \%$ dos entrevistados obtiveram o conhecimento da mesma forma. Salientase que é importante o resgate do uso e conhecimento tradicional das plantas, bem como a certificação deste com estudos científicos, para o desenvolvimento de novos fármacos, o que evidencia a importância de estudos na área das plantas medicinais, com o intuito de aproximar o saber popular do saber científico, o que oferece a população maior segurança e eficácia sobre o uso desta terapia (BADKE et al., 2011). O uso de plantas geralmente é orientado por familiares devido à falta de conhecimento dos profissionais de saúde acerca desta prática, como demostram os estudos de Rosa, Câmara e Béria (2011) realizado com médicos das ESFs de Canoas/RS, e de Schiavo, Schwambach e Colet (2017) com agentes comunitários de saúde de Ijuí/RS. Neste sentido, as Políticas do Ministério da Saúde sugerem o desenvolvimento de ações que visem à sensibilização e capacitação dos prescritores com relação ao uso seguro de plantas medicinais, além da capacitação dos agentes comunitários de saúde, com o objetivo de subsidiá-los a orientar e sanar dúvidas dos usuários sobre o assunto. Estas ações, sugeridas pelas Políticas, tem objetivo de racionalizar o uso de plantas medicinais entre as populações, perpassando esse uso de um cunho mais popular para mais científico.

Quanto à aquisição das plantas medicinais, mais da metade das entrevistadas cultivavam as plantas em sua residência (Tabela 2). Resultado semelhante foi observado no estudo de Carvalho et al. (2013) na comunidade de Várzea Garanhuns/PE, com 154 moradores, em que $71 \%$ dos entrevistados, cultivavam as plantas medicinais no quintal da própria casa, ou de vizinhos e parentes. Isso demonstra uma transmissão do conhecimento feito na própria comunidade, a partir dos pais, avós e vizinhos. Porém, o cultivo na residência pode ocasionar problemas relacionados com a identificação incorreta da planta e armazenamento impróprio, como observado no estudo de Feijó, Bueno e Ceolin (2012) bem como no presente estudo.

Quanto à identificação do material botânico coletado (Tabela 3), as famílias identificadas neste estudo foram semelhantes ao encontrado no levantamento 
Tabela 3 - Identificação da espécie, forma de uso e indicação da literatura de plantas medicinais citadas por mulheres climatéricas atendidas nas ESFs VII e VIII do município de Ijuí/RS. 2014.

\begin{tabular}{|c|c|c|c|c|c|c|c|}
\hline Família & $\begin{array}{l}\text { Nome } \\
\text { científico } \\
\text { Nome } \\
\text { popular }\end{array}$ & $\mathbf{N}$ & $\begin{array}{c}\text { Parte } \\
\text { utilizada }\end{array}$ & $\begin{array}{l}\text { Modo de } \\
\text { preparo }\end{array}$ & $\begin{array}{c}\text { Uso pelas } \\
\text { entrevistadas }\end{array}$ & $\begin{array}{l}\text { Indicação da } \\
\text { literatura }\end{array}$ & $\begin{array}{l}(\mathbf{A C}) /(\mathbf{D}) / \\
(\mathbf{P A}) / \mathbf{N A}\end{array}$ \\
\hline Alismateacea & $\begin{array}{l}\text { Echinodorus } \\
\text { grandiflorus } \\
\text { Chapéu de } \\
\text { couro }\end{array}$ & 1 & Folha & $\begin{array}{l}\text { Infusão } \\
\text { (1) }\end{array}$ & $\begin{array}{c}\text { Infecção na } \\
\text { bexiga }\end{array}$ & $\begin{array}{c}\text { Diurético, depurativo, } \\
\text { colagogo, hipotensor e } \\
\text { anti-inflamatório. }\end{array}$ & $\mathrm{AC}$ \\
\hline \multirow{3}{*}{$\begin{array}{c}\text { Apiaceae } \\
\text { (Umbeliferae) }\end{array}$} & $\begin{array}{l}\text { Foeniculum } \\
\text { vulgare Mill. } \\
\text { Funcho, erva } \\
\text { doce }\end{array}$ & 2 & Semente & $\begin{array}{l}\text { Infusão } \\
(2)\end{array}$ & $\begin{array}{l}\text { Calmante; } \\
\text { Prisão de } \\
\text { ventre }\end{array}$ & $\begin{array}{c}\text { Eliminar gases, } \\
\text { combater cólicas e } \\
\text { estimular a lactação } \\
\text { Estomáquico, } \\
\text { estimulante, } \\
\text { aromático, } \\
\text { expectorante e } \\
\text { galactogênico }^{\mathrm{D}}\end{array}$ & PA \\
\hline & $\begin{array}{l}\text { Illicium } \\
\text { verum. } \\
\text { Anis } \\
\text { estrelado }\end{array}$ & 1 & Fruto & $\begin{array}{l}\text { Maceração } \\
\text { (1) }\end{array}$ & Gripe & $\begin{array}{l}\text { Expectorante e } \\
\text { antiflatulento }\end{array}$ & $\mathrm{D}$ \\
\hline & $\begin{array}{l}\text { Apium } \\
\text { graveolens } \\
\text { Aipo }\end{array}$ & 2 & $\begin{array}{l}\text { Semente } \\
\text { e folha }\end{array}$ & $\begin{array}{l}\text { Infusão } \\
(2)\end{array}$ & $\begin{array}{c}\text { Pneumonia; } \\
\text { Intestino; Gripe }\end{array}$ & \begin{tabular}{|} 
Eliminação de gases \\
decorrentes de \\
problemas digestivos \\
e as sementes \\
apresentam efeitos \\
sedativos. Além de \\
ser expectorante e \\
febrícula ${ }^{\mathrm{B}}$ \\
Excitante, carminativo \\
e diurético
\end{tabular} & PA \\
\hline Asphodelaceae & $\begin{array}{c}\text { Aloe Vera } \\
\text { Babosa }\end{array}$ & 1 & Folha & Decocção (1) & Cicatrizante & $\begin{array}{c}\text { Uso externo: } \\
\text { Cicatrizante nos } \\
\text { casos de queimaduras } \\
\text { e ferimentos } \\
\text { superficiais na pele } \\
\text { Tratamento tópico } \\
\text { de queimaduras de } \\
1^{\mathrm{O}} \text { e } 2^{\mathrm{o}} \text { graus e como } \\
\text { coadjuvante nos casos } \\
\text { de Psoríase vulgaris } \\
\text { Anti-inflamatório, } \\
\text { emoliente, hidratante, } \\
\text { e antibacteriana } \\
\text { Purgativo, } \\
\text { estomáquico, digestivo } \\
\text { e cicatrizante }\end{array}$ & $\mathrm{AC}$ \\
\hline
\end{tabular}




\begin{tabular}{|c|c|c|c|c|c|c|c|}
\hline \multirow{10}{*}{$\begin{array}{c}\text { Asteraceae } \\
(\text { compositae })\end{array}$} & $\begin{array}{c}\text { Achyrocline } \\
\text { satureioides (Lam) } \\
\text { DC. } \\
\text { Marcela } \\
\end{array}$ & 17 & Flor & $\begin{array}{c}\text { Infusão (15), } \\
\text { Decocção } \\
\text { (2) }\end{array}$ & $\begin{array}{l}\text { Má digestão; } \\
\text { Fígado; } \\
\text { Estômago }\end{array}$ & $\begin{array}{l}\text { Digestivo estomacal, } \\
\text { hepático, intestinal e } \\
\text { diarreia }^{\mathrm{B}}\end{array}$ & $\mathrm{AC}$ \\
\hline & $\begin{array}{c}\text { Matricaria recutita } \\
\text { Camomila ou } \\
\text { maçanilha }\end{array}$ & 14 & Flor & $\begin{array}{c}\text { Infusão (12), } \\
\text { Decocção } \\
\text { (2) }\end{array}$ & $\begin{array}{l}\text { Calmante; } \\
\text { Estômago; } \\
\text { Prisão de } \\
\text { ventre }\end{array}$ & $\begin{array}{c}\text { Tônico amargo, digestivo, } \\
\text { sedativo, para facilitar } \\
\text { a eliminação de gases, } \\
\text { combater cólicas e estimular } \\
\text { o apetite } \\
\end{array}$ & $\mathrm{AC}$ \\
\hline & $\begin{array}{c}\text { Mikania glomerata } \\
\text { Spreng. } \\
\text { Guaco }\end{array}$ & 8 & Folha & $\begin{array}{c}\text { Infusão (7), } \\
\text { Decocção(1) }\end{array}$ & $\begin{array}{c}\text { Gripe; } \\
\text { Intoxicação }\end{array}$ & $\begin{array}{c}\text { Ações tônica, depurativa, } \\
\text { febrícula e peitoral, } \\
\text { estimulante do apetite e } \\
\text { antigripal }^{\mathrm{B}, \mathrm{D}} \text {; expectorante }\end{array}$ & PA \\
\hline & $\begin{array}{c}\text { Cynara scolymus } \\
\text { L. } \\
\text { Alcachofra }\end{array}$ & 2 & Folha & Infusão (2) & $\begin{array}{l}\text { Digestivo; } \\
\text { Estômago }\end{array}$ & $\begin{array}{c}\text { Ativa o funcionamento } \\
\text { da vesícula biliar } \\
\text { e protege o fígado, } \\
\text { hipocolesterolêmico }{ }^{\mathrm{B}, \mathrm{D}}\end{array}$ & $\mathrm{AC}$ \\
\hline & $\begin{array}{c}\text { Artemisea } \\
\text { absinthium L. } \\
\text { Losna ou Losma }\end{array}$ & 4 & Folha & $\begin{array}{l}\text { Infusão (3), } \\
\text { Maceração } \\
\text { (1) }\end{array}$ & $\begin{array}{l}\text { Fígado; } \\
\text { Estômago }\end{array}$ & $\begin{array}{c}\text { Perda de apetite, distúrbios } \\
\text { da digestão, do fígado e da } \\
\text { vesícula biliar }{ }^{\mathrm{B}} \\
\text { Antiespasmódica, } \\
\text { emenagoga, antiemética, } \\
\text { problemas de estômago } \\
\text { e fígado ou contra } \\
\text { vermífugos }^{\mathrm{D}} \\
\end{array}$ & $\mathrm{AC}$ \\
\hline & $\begin{array}{c}\text { Stevia rebaudiana } \\
\text { (Bertoni) } \\
\text { Stévia }\end{array}$ & 1 & Folha & Infusão (1) & Estômago & $\begin{array}{c}\text { Hipoglicemiante, } \\
\text { hipotensora, diurética e } \\
\text { cardiotônica }^{\mathrm{B}, \mathrm{D}} \\
\text { Azia }^{\mathrm{D}} \\
\end{array}$ & PA \\
\hline & $\begin{array}{c}\text { Achillea } \\
\text { millefolium } L . \\
\text { Mil folhas ou Mil } \\
\text { em rama }\end{array}$ & 1 & Folha & Infusão (1) & Estômago & $\begin{array}{c}\text { Dispepsia, diarreia, febre, } \\
\text { contra gases intestinais e } \\
\text { cálculo renal }^{\mathrm{B}} ; \\
\text { Anti-inflamatório, } \\
\text { cicatrizante, } \\
\text { antiespasmódica }^{\mathrm{D}} \\
\end{array}$ & $\mathrm{AC}$ \\
\hline & $\begin{array}{c}\text { Pluchea sagittalis } \\
\text { (Lam.) Cabrera } \\
\text { Quitoco ou Erva } \\
\text { lucera }\end{array}$ & 1 & Folha & Infusão (1) & Fígado & $\begin{array}{c}\text { Atividade antimicrobiana } \\
\text { contra o Trypanosoma } \text { cruzi }^{\mathrm{B}} \\
\text { Emenagogo, em flatulências, } \\
\text { bronquite e reumatismo }\end{array}$ & $\mathrm{D}$ \\
\hline & $\begin{array}{c}\text { Baccharis trimera } \\
\text { (Less.) DC. } \\
\text { Carqueja }\end{array}$ & 3 & Folha & Infusão (3) & $\begin{array}{l}\text { Emagrecer; } \\
\text { Digestão; } \\
\text { Estômago. }\end{array}$ & $\begin{array}{c}\text { Propriedades } \\
\text { hepatoprotetoras, } \\
\text { propriedaes digestivas, } \\
\text { antiúlcera e antiácida, efeito } \\
\text { analgésico, anti-inflamatório } \\
\text { e hipoglicemiante } \\
\text { Diurética, tônica, febrífuga } \\
\text { eupéptica }\end{array}$ & $\mathrm{AC}$ \\
\hline & $\begin{array}{c}\text { Bidens pilosa } \\
\text { Picão do Reino }\end{array}$ & 1 & Folha & Infusão (1) & $\begin{array}{l}\text { Vômito; } \\
\text { Cólicas } \\
\text { intestinais }\end{array}$ & $\begin{array}{c}\text { Atividade antibacterecida, } \\
\text { hepatoprotetora, anti- } \\
\text { inflamatória } \\
\text { Emoliente, icterícia, hepatite } \\
\text { e problemas no fígado }\end{array}$ & $\mathrm{D}$ \\
\hline
\end{tabular}


Schiavo, M. et al.

\begin{tabular}{|c|c|c|c|c|c|c|c|}
\hline Brassicaceae & $\begin{array}{c}\text { Coronopus } \\
\text { didymus (L.) SM } \\
\text { Erva de Santa } \\
\text { Maria ou mentruz }\end{array}$ & 1 & Folha & Infusão (1) & $\begin{array}{l}\text { Herpes; } \\
\text { Pâncreas }\end{array}$ & $\begin{array}{l}\text { Depurativa do sangue, } \\
\text { diurética, expectorante }{ }^{B}\end{array}$ & $\mathrm{D}$ \\
\hline Celastraceae & $\begin{array}{c}\text { Maytenus } \\
\text { ilicifolia } \\
\text { (Schrad.) Planch. } \\
\text { Cancorosa ou } \\
\text { espinheira santa }\end{array}$ & 4 & Folha & $\begin{array}{l}\text { Decocção } \\
\text { (1), Infusão } \\
\text { (3) }\end{array}$ & $\begin{array}{c}\text { Bexiga; Limpa } \\
\text { o sangue; } \\
\text { Digestivo; } \\
\text { Estômago }\end{array}$ & $\begin{array}{c}\text { Potente ação } \\
\text { antiulcerogênica }{ }^{\mathrm{B}, \mathrm{D}} ; \\
\text { Antidispéptico, } \\
\text { antiácido e protetor da } \\
\text { mucosa gástrica }^{\mathrm{C}} \\
\text { Analgésico e } \\
\text { cicatrizante }^{\mathrm{D}}\end{array}$ & PA \\
\hline Equisetaceae & $\begin{array}{c}\text { Equisetum } \\
\text { giganteum L. } \\
\text { Cavalinha }\end{array}$ & 2 & Talo & Infusão (2) & Diurético; Rins & Diurético $^{\mathrm{D}}$ & $\mathrm{AC}$ \\
\hline $\begin{array}{l}\text { Fabaceae - } \\
\text { Cercideae }\end{array}$ & $\begin{array}{c}\text { Bauhinia forficata } \\
\text { Link } \\
\text { Pata de vaca }\end{array}$ & 1 & Folha & $\begin{array}{l}\text { Decocção } \\
\text { (1) }\end{array}$ & Diabetes & $\begin{array}{l}\text { Não Encontrada na } \\
\text { Literatura pesquisada }\end{array}$ & NA \\
\hline \multirow{6}{*}{$\begin{array}{l}\text { Lamiaceae } \\
\text { (Labiatae). }\end{array}$} & $\begin{array}{c}\text { Mentha pulegium } \\
\quad L . \\
\text { Poejo ou poejinho }\end{array}$ & 4 & Folha & $\begin{array}{l}\text { Infusão (3), } \\
\text { Decocção } \\
\text { (1) }\end{array}$ & Calmante & $\begin{array}{l}\text { Afecções respiratórias } \\
\text { como expectorante, } \\
\text { estimulante do } \\
\text { apetite, perturbações } \\
\text { digestivas, espasmos } \\
\text { gastrointestinais, } \\
\text { cálculos biliares e } \\
\text { colecistite }^{\mathrm{B}, \mathrm{C}, \mathrm{D}}\end{array}$ & $\mathrm{D}$ \\
\hline & $\begin{array}{c}\text { Mentha spicata } L . \\
\text { Hortelã }\end{array}$ & 4 & Folha & Infusão (4) & $\begin{array}{l}\text { Calmante; } \\
\text { Gripe; Tosse }\end{array}$ & $\begin{array}{l}\text { Resfriados, tosse, } \\
\text { bronquite, febre, } \\
\text { calafrios }\end{array}$ & PA \\
\hline & $\begin{array}{c}\text { Melissa officinalis } \\
\text { L. } \\
\text { Melissa }\end{array}$ & 8 & Folha & $\begin{array}{l}\text { Infusão (7), } \\
\text { Decocção } \\
\text { (1) }\end{array}$ & $\begin{array}{l}\text { Calmante; } \\
\text { Nervosismo }\end{array}$ & $\begin{array}{c}\text { Calmante nos casos de } \\
\text { insônia e ansiedade } \text { a }^{\mathrm{A}, \mathrm{B}} \\
\text { Carminativo, } \\
\text { estimulante, calmante, } \\
\text { sedativo, febres e } \\
\text { resfriados }^{\mathrm{D}}\end{array}$ & $\mathrm{AC}$ \\
\hline & $\begin{array}{c}\text { Plectranthus } \\
\text { barbatus } \\
\text { Andrews } \\
\text { Falso boldo ou } \\
\text { boldo brasileiro } \\
\end{array}$ & 11 & Folha & $\begin{array}{l}\text { Maceração } \\
\text { (3), Infusão } \\
\text { (8) }\end{array}$ & $\begin{array}{c}\text { Estômago; } \\
\text { Digestão; } \\
\text { Fígado; Prisão } \\
\text { de ventre }\end{array}$ & $\begin{array}{l}\text { Controle da gastrite, } \\
\text { dispepsia, azia, mal } \\
\text { estar gástrico }{ }^{\mathrm{B}, \mathrm{D}} \text {; } \\
\text { Antidispéptico }^{\mathrm{C}}\end{array}$ & PA \\
\hline & $\begin{array}{c}\text { Mentha x piperita } \\
\text { var. Citrata } \\
\text { (Ehrh.) Briq / } \\
\text { Hortelã pimenta }\end{array}$ & 9 & Folha & $\begin{array}{l}\text { Infusão (8), } \\
\text { Decocção } \\
\text { (1) }\end{array}$ & $\begin{array}{l}\text { Calmante; } \\
\text { Estômago; } \\
\text { Insônia; Dor } \\
\text { de garganta; } \\
\text { Digestivo }\end{array}$ & $\begin{array}{c}\text { Antiespasmódico e } \\
\text { antiflatulento }^{\mathrm{C}, \mathrm{D}} \\
\text { Estomacal, estimulante } \\
\text { e vermífugo }^{\mathrm{D}}\end{array}$ & PA \\
\hline & $\begin{array}{l}\text { Ocimum } \\
\text { basilicum L. } \\
\text { Alfavaca }\end{array}$ & 1 & Folha & Infusão (1) & $\begin{array}{l}\text { Fígado; } \\
\text { Estômago }\end{array}$ & $\begin{array}{c}\text { Problemas digestivos } \\
\text { (estômago, hepático, } \\
\text { vesícula biliar e gases } \\
\text { intestinais) }^{\mathrm{B}}\end{array}$ & $\mathrm{AC}$ \\
\hline
\end{tabular}


Conhecimento sobre plantas medicinais por mulheres em processo de envelhecimento

\begin{tabular}{|c|c|c|c|c|c|c|c|}
\hline Lythraceae & $\begin{array}{c}\text { Punica granatum } \\
\text { L. } \\
\text { Romã }\end{array}$ & 1 & Casca & Infusão (1) & Intestino & $\begin{array}{c}\text { Inflamações e infecções da } \\
\text { mucosa da boca e faringe } \\
\text { como anti-inflamatório e } \\
\text { antisséptico }\end{array}$ & $\mathrm{D}$ \\
\hline Malvaceae & $\begin{array}{c}\text { Malva sylvestris } \\
\text { L. } \\
\text { Malva }\end{array}$ & 1 & Folha & Infusão (1) & Infecção & $\begin{array}{c}\text { O uso externo é para } \\
\text { bochechos e gargarejos, contra } \\
\text { inflamações e afecções da boca } \\
\text { e garganta. Para bronquite } \\
\text { crônica e outras afecções } \\
\text { do trato respiratório } \\
\text { interno: Uso expectorante. Uso } \\
\text { externo: anti-inflamatório e } \\
\text { antisséptico da cavidade oral }\end{array}$ & $\mathrm{AC}$ \\
\hline Moraceae & $\begin{array}{l}\text { Morus alba } L . \\
\text { Amoreira branca }\end{array}$ & 1 & Folha & Infusão (1) & $\begin{array}{c}\text { Calorões } \\
\text { menopausa }\end{array}$ & $\begin{array}{c}\text { Não Encontrada na Literatura } \\
\text { pesquisada }\end{array}$ & NA \\
\hline \multirow{4}{*}{ Myrtaceae } & $\begin{array}{l}\text { Syzygium cumini } \\
\text { (L.) Skeels. } \\
\text { Jambolão }\end{array}$ & 1 & Folha & Infusão (1) & Diabetes & $\begin{array}{l}\text { Hipoglicemiante } \\
\text { Adstringente } \\
\text { Ad }\end{array}$ & $\mathrm{AC}$ \\
\hline & $\begin{array}{l}\text { Campomanesia } \\
\text { affinis } \\
\text { Guabiroba ou } \\
\text { guavirova }\end{array}$ & 2 & Folha & Infusão (2) & $\begin{array}{l}\text { Reduz o } \\
\text { colesterol } \\
\text { e ajuda } \\
\text { emagrecer }\end{array}$ & $\begin{array}{c}\text { Não Encontrada na Literatura } \\
\text { pesquisada }\end{array}$ & NA \\
\hline & $\begin{array}{c}\text { Eugenia uniflora } \\
\text { Pitanga }\end{array}$ & 1 & Folha & Infusão (1) & Intestino & $\begin{array}{c}\text { Diarreia, febre e verminoses } \\
\text { infantis } \\
\text { Diurético, antirreumático, } \\
\text { antidiarreico, febrífuco e } \\
\text { sialagogo }{ }^{\mathrm{D}}\end{array}$ & $\mathrm{AC}$ \\
\hline & $\begin{array}{c}\text { Eucaliptus } \\
\text { globulus Labill. } \\
\text { Eucalipto, } \\
\text { eucalipto limão }\end{array}$ & 1 & Folha & Infusão (1) & Gripe & $\begin{array}{c}\text { Por inalação age como } \\
\text { expectorante, gripe e } \\
\text { congestão nasal } \\
\text { Antisséptico, estomacal e } \\
\text { febrífugo }{ }^{\mathrm{D}}\end{array}$ & $\mathrm{AC}$ \\
\hline Passifloraceae & $\begin{array}{c}\text { Passiflora alata } \\
\text { Curtis } \\
\text { Maracujá } \\
\end{array}$ & 1 & Folha & Decocção(1) & Estresse & Hipnótico e sedativo $^{\mathrm{D}}$ & $\mathrm{AC}$ \\
\hline Phyllantaceae & $\begin{array}{l}\text { Phyllanthus } \\
\text { niruri L. } \\
\text { Quebra-pedra }\end{array}$ & 4 & Folha & $\begin{array}{l}\text { Infusão (3), } \\
\text { Maceração } \\
\text { (1) }\end{array}$ & $\begin{array}{c}\text { Diurético; } \\
\text { Pedras nos } \\
\text { rins; Rins }\end{array}$ & $\begin{array}{c}\text { Diurético, desobstruinte, } \\
\text { moléstias do fígado e vias } \\
\text { urinárias }^{\mathrm{D}}\end{array}$ & $\mathrm{AC}$ \\
\hline Piperaceae & $\begin{array}{c}\text { Piper } \\
\text { umbellatum } L . \\
\text { Pariparoba } \\
\end{array}$ & 2 & Folha & \begin{tabular}{l} 
Decocção \\
$(1)$, Infusão \\
\multicolumn{1}{c}{$(1)$} \\
\end{tabular} & $\begin{array}{l}\text { Fígado; } \\
\text { Intestino }\end{array}$ & $\begin{array}{c}\text { Diurética e usada para doenças } \\
\text { do fígado e da vesícula }{ }^{\mathrm{B}, \mathrm{D}}\end{array}$ & PA \\
\hline Plantaginaceae & $\begin{array}{c}\text { Plantago major } \\
\text { L. } \\
\text { Tansagem }\end{array}$ & 3 & Folha & Infusão (3) & Antibiótico & $\begin{array}{c}\text { Após higienização, aplicar o } \\
\text { infuso com auxílio de algodão } \\
\text { sobre o local afetado, três } \\
\text { vezes ao dia. Fazer bochechos } \\
\text { ou gargarejos três vezes ao } \\
\text { dia }^{\mathrm{C}} \\
\text { Tônico, febrífugo, } \\
\text { adstringente, anti-inflamatório } \\
\text { de mucosas e vias } \\
\text { respiratórias }^{\mathrm{D}}\end{array}$ & PA \\
\hline
\end{tabular}




\begin{tabular}{|c|c|c|c|c|c|c|c|}
\hline $\begin{array}{c}\text { Poaceae } \\
\text { (Gramineae) }\end{array}$ & $\begin{array}{c}\text { Cymbopogon } \\
\text { citratus (DC.) Stapf } \\
\text { Cidreira, capim- } \\
\text { cidreira, erva- } \\
\text { cidreira } \\
\end{array}$ & 8 & Folha & $\begin{array}{c}\text { Infusão (7), } \\
\text { Decocção (1) }\end{array}$ & $\begin{array}{l}\text { Calmante; } \\
\text { Tosse; } \\
\text { Pressão alta }\end{array}$ & $\begin{array}{c}\text { Cólicas intestinais e } \\
\text { uterinas, nervosismo } \\
\text { Antiespasmódico, } \\
\text { ansiolítico e sedativo leve } \\
\text { C,D }\end{array}$ & PA \\
\hline Polygonaceae & $\begin{array}{l}\text { Polygonum } \\
\text { hydropiperoides } \\
\text { Michx. } \\
\text { Erva de bicho }\end{array}$ & 1 & Folha & Infusão (1) & $\begin{array}{l}\text { Herpes; } \\
\text { Pâncreas }\end{array}$ & $\begin{array}{l}\text { Internamente usada para } \\
\text { diarreia e parasitoses } \\
\text { intestinais e externamente } \\
\text { usada no tratamento de } \\
\text { erisipelas e hemorroidas }^{\mathrm{B}, \mathrm{D}} \\
\end{array}$ & $\mathrm{D}$ \\
\hline Rosaceae & $\begin{array}{c}\text { Eriobotrya japonica } \\
\text { (Thunb.) } \\
\text { Ameixa do inverno, } \\
\text { ameixa amarela }\end{array}$ & 1 & $\begin{array}{l}\text { Flor e } \\
\text { folha }\end{array}$ & Infusão (1) & Gripe & $\begin{array}{l}\text { Diurética, laxativa, } \\
\text { emoliente, tosses e } \\
\text { resfriados }^{\mathrm{D}}\end{array}$ & $\mathrm{AC}$ \\
\hline \multirow{3}{*}{ Rutaceae } & $\begin{array}{c}\text { Citrus aurantium L. } \\
\text { Laranja }\end{array}$ & 4 & $\begin{array}{c}\text { Folha e } \\
\text { casca }\end{array}$ & $\begin{array}{l}\text { Decocção } \\
\text { (2), Infusão } \\
\text { (2) }\end{array}$ & Gripe & $\begin{array}{c}\text { Ansiolítico e sedativo } \\
\text { leve } \mathrm{e}^{\mathrm{C}, \mathrm{D}} \\
\begin{array}{c}\text { Gripes, expectorante e } \\
\text { cefalgias }^{\mathrm{D}}\end{array} \\
\end{array}$ & $\mathrm{AC}$ \\
\hline & $\begin{array}{c}\text { Citrus reticulata } \\
\text { Bergamota }\end{array}$ & 1 & Folha & Decocção (1) & Gripe; Febre & $\begin{array}{l}\text { Não Encontrada na } \\
\text { Literatura pesquisada. }\end{array}$ & NA \\
\hline & $\begin{array}{l}\text { Citrus aurantifolia } \\
\text { Lima ou lima pérsia }\end{array}$ & 1 & Folha & Decocção (1) & Gripe & $\begin{array}{l}\text { Não Encontrada na } \\
\text { Literatura pesquisada. }\end{array}$ & NA \\
\hline Sapotaceae & $\begin{array}{l}\text { Sideroxylon } \\
\text { obstusifolium } \\
\text { Coronília ou } \\
\text { quixaba }\end{array}$ & 1 & Casca & Decocção (1) & Coração & $\begin{array}{c}\text { A casca tem poder } \\
\text { tônico, adstringente, anti- } \\
\text { inflamatório e antidiabética }\end{array}$ & $\mathrm{D}$ \\
\hline Smilacaceae & $\begin{array}{c}\text { Smilax japipanga } \\
\text { Griseb. } \\
\text { Japecanga, } \\
\text { salsaparrilha ou raiz } \\
\text { da China }\end{array}$ & 1 & $\begin{array}{l}\text { Casca e } \\
\text { talo }\end{array}$ & Decocção (1) & $\begin{array}{c}\text { Purificador } \\
\text { do sangue }\end{array}$ & $\begin{array}{l}\text { Purificador do sangue, } \\
\text { diurético e diaforético }{ }^{\mathrm{B}}\end{array}$ & $\mathrm{AC}$ \\
\hline \multirow[t]{2}{*}{ Verbenaceae } & $\begin{array}{l}\text { Aloysia triphylla } \\
\text { Royle } \\
\text { Cidró ou erva } \\
\text { cidreira }\end{array}$ & 6 & Folha & $\begin{array}{c}\text { Infusão (5), } \\
\text { Decocção (1) }\end{array}$ & $\begin{array}{l}\text { Gripe; } \\
\text { Calmante; } \\
\text { Diminui a } \\
\text { pressão }\end{array}$ & $\begin{array}{c}\text { Resfriados febris, digestivo, } \\
\text { estimulante e calmante } \\
\text { Calmante, palpitações, } \\
\text { flatulência e } \\
\text { antiespasmódica }^{\mathrm{D}} \\
\end{array}$ & PA \\
\hline & $\begin{array}{l}\text { Lippia alba (Mill.) } \\
\text { N.E.Br. } \\
\text { Sálvia, erva-cidreira } \\
\text { de arbusto e lípia }\end{array}$ & 2 & Folha & Infusão (2) & Gripe & $\begin{array}{l}\text { Ansiolítico, sedativo } \\
\text { leve, antiespasmódico e } \\
\text { antidispéptico }^{\mathrm{C}} \\
\text { Transtornos metabólicos } \\
\text { e endócrinos (anemia, } \\
\text { hipertioidismo, } \\
\text { dismenorreia, depressão } \\
\text { nervosa, diarreia, colite e } \\
\text { parasitoses intestinais) }^{\mathrm{D}}\end{array}$ & $\mathrm{D}$ \\
\hline
\end{tabular}

* (AC) Acordo com a literatura/ (D) Desacordo com a literatura/ (PA) Parcialmente Adequado/ (NA) Não encontrada na literatura pesquisada.

A- Simões et al. (2007).

B- Lorenzi e Matos (2008).

C - ANVISA (2011).

D-Grandi (2014).

Fonte: Autores. 
botânico realizado por Battisti et al. (2013), em que foram identificadas 128 espécies distribuídas em 53 famílias botânicas no qual as famílias com maior número de espécies citadas foram Asteraceae (19), Lamiaceae (16) e Myrtaceae (6). Entretanto, no estudo citado, o número de famílias e espécies encontradas foi superior ao encontrado em nossa pesquisa. Isso se justifica pelo fato desse estudo os autores realizarem uma técnica de amostragem conhecida como "snow ball", uma amostragem intencional na qual os sujeitos envolvidos são selecionados a partir de indicações feitas pelos entrevistados da comunidade e pelos próprios informantes. A partir do contato inicial com a comunidade, um primeiro especialista é reconhecido, que passa a indicar outro especialista e assim sucessivamente, envolvendo todos os especialistas da comunidade, até que o ciclo se feche e novos especialistas não sejam mais apontados, ou seja, os participantes foram pessoas que tinham um grande conhecimento sobre as plantas medicinais. Por outro lado, em nosso estudo foram entrevistadas as mulheres, enquadradas nos critérios de inclusão, independente do seu conhecimento e uso de plantas medicinais.

Silva et al. (2012), em seu trabalho realizado no município de Mossoró/RN, com 50 moradores, cujo objetivo foi avaliar os riscos em potencial do uso indiscriminado de plantas medicinais, verificaram que o uso empírico com a ausência da comprovação adequada das plantas medicinais pode gerar riscos como intoxicação, reações alérgicas e ineficácia do tratamento. Isso pode estar relacionado ao erro na identificação das espécies ou à forma de cultivo, colheita, armazenagem e preparo. No mesmo estudo observou-se que $82 \%$ dos entrevistados acreditavam que as plantas são completamente inofensivas, mais seguras que os medicamentos convencionais, que não apresentam contraindicação e reação adversa. No presente estudo, metade das mulheres relatou que as plantas não fazem mal à saúde, o que indica carência de informação nesta população. Esse fato pode estar relacionado à crença de que "o que é natural não faz mal", o que é um grande equívoco, além de representar risco à saúde das usuárias, pois segundo França et al. (2008), as plantas são constituídas de substâncias farmacologicamente ativas, que oferecem riscos e toxicidade à saúde da população.

Da mesma forma que no presente estudo, Andrade et al. (2012) identificaram as folhas como a parte da planta mais utilizada pelos participantes do seu estudo, cujo objetivo foi analisar sob o ponto de vista etnobotânico, a utilização de plantas medicinais na comunidade Várzea Comprida dos Oliveiras/PB com 40 participantes, sendo as partes mais citadas as folhas, casca e raiz respectivamente. Além disso, 52\% dos entrevistados por Battisti et al. (2013) relataram utilizar para o preparo dos chás as folhas, através de infusão, semelhante a este estudo. Segundo os autores, o que justifica o uso das folhas ser maior, é o fato de essas serem de fácil coleta e estarem disponíveis no decorrer do ano, não dependendo de sazonalidade.

Com relação às plantas mais utilizadas e sua indicação (Tabela 3), há concordância entre as indicações populares e a literatura consultada sobre a Achyrocline satureioides (Lam) DC. (marcela). Destaca-se que não há contraindicações de seu uso na bibliografia pesquisada (LORENZI; MATOS, 2008).

De acordo com Lorenzi e Matos (2008), a Matricaria recutita (camomila) apresenta ação sobre cólicas de crianças a partir da aplicação de compressas mornas sobre o abdômen, além disso, é digestivo, sedativo e facilita a eliminação de gases (GRANDI, 2014). Assim, esta planta também apresentou concordância entre as indicações populares e o efeito comprovado na literatura pesquisada. Em estudo realizado por Lima, Pires e Vieira (2014), a camomila também foi utilizada pelos entrevistados como calmante, semelhante a este estudo, estando de acordo com a literatura. Isso porque esta planta tem uso bastante difundido, o que facilita o seu uso adequado.

Destaca-se que foram consultadas fontes secundárias de informação, como livros e manuais, os quais se constituem em ferramentas para o acesso de informações sobre plantas medicinais pelos profissionais de saúde brasileiros. No entanto, a não realização de pesquisa individualizada das plantas medicinais em ensaios clínicos e revisões sistemáticas trata-se de uma limitação do estudo.

Plectranthus barbatus Andrews (falso boldo ou boldo brasileiro) pode ser usado para dispepsia e hipotensão. Porém, quando utilizado em doses elevadas ou períodos prolongados pode causar irritação gástrica e apresentar efeito cardioativo, o qual é contraindicado para gestantes, lactantes, crianças, pessoas com hipertensão, hepatites e obstrução das vias biliares (GRANDI, 2014). Nesse estudo, esta planta está parcialmente adequada, pois algumas mulheres utilizavam para prisão de ventre e problemas hepáticos, não estando de acordo com a bibliografia consultada. Além disso, a mesma possui várias contraindicações, o que provavelmente não é 
de conhecimento desta população, sendo necessárias intervenções sobre o uso da mesma. Vale ressaltar, que o conhecimento de contraindicações e riscos não foi pesquisado, tratando-se de outra limitação deste estudo.

Mentha x piperita var. citrata (hortelã pimenta) é usada popularmente em distúrbios digestivos como náuseas e cólicas, em diarreias, resfriados, dores de cabeça, musculares, de garganta e dentes. Dados químicos e farmacológicos comprovam, a partir das folhas, atividade colerética, antiinflamatória e antiúlcera (SIMÕES et al., 2007). Não deve ser utilizada: por gestantes, crianças de pouca idade, pacientes com obstruções biliares, danos hepáticos e durante a lactação (GRANDI, 2014). No presente estudo, esta planta está parcialmente adequada com relação às indicações citadas pelas entrevistadas, pois uma mulher utilizava para insônia. Destaca-se que nem todas as plantas apresentam o efeito esperado por quem as utiliza, ou seja, a planta pode não apresentar o efeito para o qual foi popularmente indicada, como pode ter ocorrido com esta entrevistada. Deve-se ter cuidado, pois pode ocorrer aumento de dose a procura do efeito esperado, ocorrendo intoxicação, entre outros riscos.

Especificamente sobre plantas utilizadas para tratamento da menopausa, foram citadas a Morus alba L. (amoreira branca) e a Calendula officinalis L. (calêndula), ambas usadas para diminuir os calorões e outros sintomas relacionados com a menopausa. A primeira planta citada não foi encontrada na literatura pesquisada, e a outra não há até o momento comprovações da finalidade citada pela entrevistada, sendo recomendada pelo Ministério da Saúde para inflamações, lesões, contusões e queimadura, além de ser usada como antiinflamatório e cicatrizante (ANVISA, 2011). A Morus nigra L. (amoreira preta) e a Morus alba L. (amoreira branca) foram citadas no estudo de Vendruscolo e Mentz (2006) para minimizar os sintomas do climatério, porém, Bitencourt, Langeloh e Dallegrave (2007) concluíram que o extrato hidroalcoólico e a infusão das folhas desta planta não apresentavam atividade estrogênica. Estes dados demonstram que a utilização de plantas medicinais para tratamento dos sintomas da menopausa ainda é incipiente, e pode estar relacionado com a falta de estudos que comprovem a eficácia de plantas para este fim. Além disso, as participantes não citaram a isoflavona de soja (Glycine max (L.) Merr.) e cimicífuga (Actaea racemosa L.), que está indicada segundo a ANVISA (2014), para auxiliar no alívio dos sintomas do climatério, provavelmente por falta de conhecimento sobre a sua indicação e pelo fato do município não disponibilizar fitoterápicos a base destas plantas na sua relação de medicamentos essenciais.

Das plantas citadas na Tabela 3, para cinco espécies não foram encontradas nas bibliografias pesquisadas informações que fossem ao encontro do uso indicado. Isso revela como a população estudada apresenta carência de informação sobre a indicação correta das plantas medicinais, ou seja, muitas mulheres utilizavam de forma incorreta ou pelo menos, não comprovada por estudos científicos. Ao contrário da indicação, todas as plantas medicinais citadas pelas entrevistadas foram identificadas por elas, pelos nomes populares corretos. Com isso, percebe-se o bom conhecimento popular sobre a identificação das plantas medicinais relatado pelas entrevistadas.

Destaca-se, neste contexto, o conhecimento e o uso de plantas medicinais pelas mulheres usuárias das ESFs do presente estudo. Estes dados reforçam a importância da discussão e incentivo do uso de plantas medicinais pelos serviços de atenção primária a saúde (APS), especialmente as equipes de ESF que se constituem na porta de entrada dos serviços de saúde e possuem sob sua responsabilidade um determinado território, as quais necessitam conhecer as condições de saúde e sócio-culturais de seus usuários. Nesse sentido, infere-se que o uso de plantas medicinais na APS constitui-se em uma ferramenta de cuidado, uma vez que permite a promoção da saúde, pois envolve o resgate de valores culturais, facilita o vínculo equipecomunidade, aproximam profissionais e usuários; estimula a autonomia do cuidado, o desenvolvimento local, a intersetorialidade e a participação comunitária (ANTONIO; TESSER; MORETTI-PIRES, 2013).

$\mathrm{Na}$ revisão sobre os programas e as ações da APS envolvendo plantas medicinais Antonio, Tesser e Moretti-Pires (2013) identificaram atividades educativas que envolveram tanto a comunidade quanto os profissionais de saúde e destacam que a educação permanente e a popular em relação à fitoterapia proporcionam democratização dos saberes, diálogo, aprendizado, orientação, escuta e enfrentamento criativo dos problemas de saúde cotidianos dos serviços, resultando em melhoria da qualidade do cuidado. Ainda, os autores inferem que a interação entre diferentes saberes (profissionais e comunidade) apresentam-se como o caminho para o fortalecimento e efetivação de políticas públicas relacionadas a 
plantas medicinais, que não busca unicamente a redução de custos com saúde, validação e certificação de produtos fitoterápicos tecnicamente elaborados, mas, especialmente, a promoção da saúde, a escuta qualificada, a solidariedade e a emancipação social.

Uma alternativa na APS para a correta utilização das plantas medicinais pode ser a implementação de Farmácias Vivas, pois através delas, hortas de plantas medicinais poderiam ser instaladas próximas às unidades de ESF e centros comunitários fornecendo plantas medicinais gratuitamente para a população, que poderia compor sua farmácia caseira com plantas certificadas por botânicos, e ainda as mulheres poderiam ser instruídas por um profissional da saúde sobre as melhores práticas de sua utilização, forma de preparo, posologia, armazenamento e riscos de interações entre plantas e medicamentos (MATOS, 1998). O que ressalta a importante da participação ativa dos profissionais da APS em relação à discussão, capacitação e integração de práticas como a fitoterapia, através de uma relação dialética entre o conhecimento popular e o científico (FONTENELE et al., 2013).

Além disso, destaca-se que muitas plantas apresentam indicações de uso popular, mas estas não são comprovadas cientificamente e pela literatura limitada identificam-se plantas sem indicações documentadas. Ainda, estudos in vitro e em animas não podem ser utilizados de base para o uso terapêutico de plantas medicinais em seres humanos, pois são estudos com característica exploratória para avaliar a atividade farmacológica e toxicológica destas plantas, antes que estas sejam testadas por meio de ensaio clínico em seres humanos.

Observou-se que a maioria das entrevistadas faz uso de algum tipo de planta medicinal para cuidar da saúde, com conhecimento passado de geração em geração. Foram citadas diferentes espécies de plantas medicinais, sendo que todas as plantas foram identificadas, pelas entrevistadas, denominando-as conforme nome popular correto, o que demonstra conhecimento das participantes quanto à identificação das espécies vegetais. Por outro lado, no que se refere à indicação de uso, algumas espécies estão em desacordo com a literatura, o que indica a necessidade de orientação para as usuárias do serviço público de saúde em relação ao uso e a toxicidade das plantas medicinais.

\section{Referências}

ALVES, G. S. P.; POVH, J. A. Estudo etnobotânico de plantas medicinais na comunidade de Santa Rita, Ituiutaba /MG. Revista Biotemas, Florianópolis, v. 26, n. 3, p. 231-242, 2013.

ANDRADE, S. E. O.; MARACAJÁ, P. B.; SILVA, R. A.; FREIRES, G. F.; PEREIRA, A .M. Estudo etnobotânico de plantas medicinais na comunidade Várzea Comprida dos Oliveiras, Pombal, Paraíba, Brasil. Revista Verde de Agroecologia e Desenvolvimento Sustentável, Paraíba, v. 7, n. 3, p. 46-52, 2012.

ANTONIO, G. D.; TESSER, C. D.; MORETTI-PIRES, R. O. Contribuições das plantas medicinais para o cuidado e a promoção da saúde na atenção primária. Interface, Botucatu, v. 17, n. 46, p. 615-33, 2013.

ANVISA. Agência Nacional de Vigilância Sanitária. Formulário de fitoterápicos da farmacopéia brasileira. Brasília, 2011.

ANVISA. Agência Nacional de Vigilância Sanitária. Instrução Normativa $n^{\circ} 2$, de 13 de maio de 2014. Publica a "Lista de medicamentos fitoterápicos de registro simplificado" e a "Lista de produtos tradicionais fitoterápicos de registro simplificado". Disponível em: $\quad<$ http://bvsms.saude.gov.br/bvs/saudelegis/ anvisa/2014/int0002_13_05_2014.pdf >. Acesso em: 5 jul. 2017.

BADKE, M. R.; BUDÓ, M. L. D.; SILVA, F. M.; RESSEL, L. B. Plantas medicinais: o saber sustentado na prática do cotidiano popular. Escola Anna Nery, Rio de Janeiro, v. 15, n. 1, p. 132-139, 2011.

BATTISTI, C.; GARLET, T. M. B.; ESSI, L.; HORBACH，R. K.; ANDRADE，A.; BADKE，M. R. Plantas medicinais utilizadas no município de Palmeira das Missões, RS, Brasil. Revista Brasileira de Biociência, Porto Alegre, v. 11, n. 3, p. 338-48, 2013.

BERTOLDI, A. D.; BARROS, A. J. D.; HALLAL, P. C.; LIMA, R. C. Utilização de medicamentos em adultos: prevalência e determinantes individuais. Revista de Saúde Pública, São Paulo, v. 38, n. 2, p. 228-38, 2004.

BITENCOURT, A. P. N.; LANGELOH, A.; DALLEGRAVE, E. Avaliação da atividade fitoestrogênica do extrato hidroalcoólico e da infusão das folhas de Morus nigra L. Acta Scientiae Veterinarie, Porto Alegre, v. 35, n. 3, p. 403-404, 2007. 
BRASIL. Ministério da Saúde. Secretaria de Atenção à Saúde. Departamento de Atenção Básica. Política nacional de práticas integrativas e complementares no sistema único de saúde (PNPIC). Brasília, 2006a. Disponível em: <http://bvsms.saude.gov.br/bvs/ publicacoes/pnpic.pdf>. Acesso em: 15 fev. 2014.

BRASIL. Ministério da Saúde. Secretaria de Atenção à Saúde. Departamento de Ações Programáticas Estratégicas. Manual de atenção à mulher no climatério/menopausa. Brasília, 2008. (Normas e Manuais Técnicos, Série A).

BRASIL. Ministério da Saúde. Secretaria de Ciência, Tecnologia e Insumos Estratégicos. Departamento de Assistência Farmacêutica. Política nacional de plantas medicinais e fitoterápicos (PNPMF). Brasília, 2006b. Disponível em: <http://www. mda.gov.br/sitemda/sites/sitemda/files/user arquivos_64/Pol\%C3\%ADtica_Nacional_de_Plantas_ Medicinais_e_Fitoter\%C3\%Alpicos.pdf $>$. Acesso em: 15 fev. 2014.

CARVALHO, J. S. B.; MARTINS, J. D. L.; MENDONÇA, M. C. S.; LIMA, L. D. Uso popular das plantas medicinais na comunidade da Várzea, Garanhuns-PE. Revista de Biologia e Ciências da Terra, São Cristóvão, v. 13, n. 2, p. 58-65, 2013.

DELL'ANTONIO, L. R.; COELHO, L. S.; SOUZA, C. B.; SACRAMENTO, H. T.; ZANDONADE, E.; AMORIM, M. H. C. O uso de plantas medicinais por mulheres com diagnóstico de câncer de mama em um programa de reabilitação. Revista Brasileira de Pesquisa em Saúde, Vitória, v. 17, n. 4, p. 85-97, out./ dez. 2015.

FEIJÓ, A. M.; BUENO, M. E. N.; CEOLIN, T. Plantas medicinais utilizadas por idosos com diagnóstico de diabetes mellitus no tratamento dos sintomas da doença. Revista Brasileira de Plantas Medicinais, Botucatu, v. 14, n. 1, p. 50-6, 2012.

FONTENELE, R. P.; SOUZA, D. M. P.; CARVALHO, A. L. M.; OLIVEIRA, F. A. Fitoterapia na atenção básica: olhares dos gestores e profissionais da estratégia saúde da família de Teresina (PI), Brasil. Ciência \& Saúde Coletiva, Rio de Janeiro, v. 18, n. 8, p. 23852394, 2013.

FRANÇA, I. S. X.; SOUZA, J. A.; BATISTA, R. S.; BRITTO, V. R. S. Medicina popular: benefícios e malefícios das plantas medicinais. Revista Brasileira de Enfermagem, Brasília, v. 61, n. 2, p. 201-8, 2008.
GONÇALVES, A. K. S.; CANÁRIO, A. C. G.; CABRAL, P. U. L.; SILVA, R. A. H.; SPYRDES, M. H. C.; GIRALDO, P. C.; ELEUTÉRIO JUNIOR, J. Impacto da atividade física na qualidade de vida de mulheres de meia idade: estudo de base populacional. Revista Brasileira de Ginecologia e Obstetrícia, Rio de Janeiro, v. 33, n. 12, p. 408-13, 2011.

GRANDI, T. S. M. Tratado das plantas medicinais: mineiras, nativas e cultivas. Belo Horizonte: Adaequatio Estúdio, 2014.

HOGA, L. A. K. Illness care at home or in health institutions: the decision process in a low income community. Revista Latino-Americana de Enfermagem, Ribeirão Preto, v. 16, n. 1, p. 115-121, 2008.

KARAM, K. F. A mulher na agricultura orgânica e em novas ruralidades. Estudos Feministas, Florianópolis, v. 12, n. 1, p. 303-320, 2004.

LIMA, R. A.; PIRES, S. S.; VIEIRA, N. G. A. A educação ambiental e o uso de plantas medicinais utilizadas pela população do distrito de União Bandeirante-Rondônia. REGET, Santa Maria, v. 18, n. 4, p. 1351-60, 2014.

LIMA, R. A.; PIRES, S. S.; VIEIRA, N. G. A. A educação ambiental e o uso de plantas medicinais utilizadas pela população do distrito de União

Bandeirante-Rondônia. REGET, Santa Maria, v. 18, n. 4, p. 1351-60, 2014.

LORENZI, H.; MATOS, F. J. Plantas medicinais no Brasil: nativas e exóticas. 2. ed. 2008.

MATOS, A. B. T. M.; MATOS, L. T. M. B.; BRITO, N. M. M. B. Uso empírico de plantas medicinais por mulheres. Revista Paraense de Medicina, Belém, v. 22, n. 4, p. 49-52, 2008.

MATOS, F. J. A. Farmácias vivas. Fortaleza: Universidade Federal do Ceará, 1998.

MENTZ, L. A.; BORDIGNON, S. A. L. Nomenclatura botânica, classificação e identificação de plantas medicinais. In: SIMÕES, C. M. O.; SCHENKEL, E. P.; GOSMANN, G.; MELO, J. C. P.; MENTZ, L. A.; PETROVICK, P. R. (Org.). Farmacognosia da planta ao medicamento. Porto Alegre: UFRGS; Florianópolis: UFSC, 2004. p. 211-227. 
OLIVEIRA, D. M. S.; LUCENA, E. M. P. O uso de plantas medicinais por moradores de Quixadá-Ceará. Revista Brasileira de Plantas Medicinais, Campinas, v. 17, n. 3, p. 107-412, 2015.

PEREIRA, J. B. A.; RODRIGUES, M. M.; MORAIS, I. R.; VIEIRA, C. R. S.; SAMPAIO, J. P. M.; MOURA, M. G.; DAMASCENO, M. F. M.; SILVA, J. N.; CALOU, I. B. F.; DEUS, F. A.; PERON, A. P.; ABREU, M. C.; MILITÃO, G. C. G.; FERREIRA, P. M. P. O papel terapêutico do Programa Farmácia Viva e das plantas medicinais o centro-sul piauiense. Revista Brasileira de Plantas Medicinais, Campinas, v. 17, n. 4, p. 550-561, 2015.

PETRY, K.; ROMAN JÚNIOR, W. A. Viabilidade de implantação de fitoterápicos e plantas medicinais no Sistema Único de Saúde do município de Três Passos/ RS. Revista Brasileira de Farmácia, Rio de Janeiro, v. 93, n. 1, p. 60-67, 2012.

PILlA, M. A.; AMOROZO, M. C. M.; FURLAN, A. Obtenção e uso das plantas medicinais no distrito de Martim Francisco, Município de Mogi-Mirim, SP, Brasil. Revista Acta Botânica Brasílica, Belo Horizonte, v. 20, n. 4, p. 789-802, 2006.

RATES, S. M. K. Promoção do uso racional de fitoterápicos: uma abordagem no ensino de Farmacognosia. Revista Brasileira de Farmacognosia, Maringá, v. 11, n. 2, p. 57-69, 2001.

ROCHA, M. D. H. A.; ROCHA, P. A. Do climatério à menopausa. Revista Científica do ITPAC, Araguaína, v. 3, n. 1, p. 24-7, 2010.

ROSA, C.; CÂMARA, S. G.; BÉRIA, J. U. Representações e intenção de uso da fitoterapia na atenção básica à saúde. Ciência \& Saúde Coletiva, Rio de Janeiro, v. 16, n. 1, p. 311-318, 2011.

ROSA, P. L. F. S.; HOGA, L. A. K.; SANTANA, M. F.; SILVA, P. A. L. Uso de plantas medicinais por mulheres negras: estudo etnográfico em uma comunidade de baixa renda. Revista da Escola de Enfermagem da USP, São Paulo, v. 48, n. esp., p. 46-53, 2014.

SCHIAVO, M.; SCHWAMBACH, K. H.; COLET, C. F. Conhecimento sobre plantas medicinais e fitoterápicos de agentes comunitários de saúde de Ijuí/RS. Revista Online de Pesquisa Cuidado é Fundamental, Rio de Janeiro, v. 9, n. 1, p. 57-63, jan./mar. 2017.
SCHWAMBACH, K. H.; AMADOR, T. A. Estudo da utilização de plantas medicinais e medicamentos em um município do sul do Brasil. Latin American Journal of Pharmacy, La Plata, v. 26, n. 4, p. 602-8, 2007.

SILVA, N. C. B.; REGIS, A. C. D.; ESQUIBEL, M. A.; SANTOS, J. E. S.; ALMEIDA, M. Z. Uso de plantas medicinais na comunidade quilombola da Barra II Bahia, Brasil. Boletín Latinoamericano y del Caribe de Plantas Medicinales y Aromáticas, Santiago, v. 11, n. 5, p. 435-53, 2012.

SIMÕES, C. M. O.; SCHENKEL, E. P.; GOSMANN, G.; MELlO, J. C. P.; MENTZ, L. A.; PETROVICK, P. R. (Org.). Farmacognosia: da planta ao medicamento. 6. ed. Porto Alegre: UFRGS, 2007.

SOUZA, F. C.; OLIVEIRA, E. N. A.; SANTOS, D. C.; OlIVEIRA, F. A. A.; MORI, E. Uso de plantas medicinais (fitoterápicos) por mulheres da cidade de Icó-CE. Biofar: Revista de Biologia e Farmácia, Campina Grande, v. 5, n. 1, p. 161-170, 2011.

VENDRUSCOLO, G. S.; MENTZ, L. A. Estudo da concordância das citações de uso e importância das espécies e famílias utilizadas como medicinais pela comunidade do bairro Ponta Grossa, Porto Alegre, RS, Brasil. Acta Botânica Brasílica, São Paulo, v. 20, n. 2, p. 367-82, 2006.

Recebido em: 21 set. 2016 Aceito em: 30 jun. 2017 
Schiavo, M. et al. 